\title{
Bronchopulmonary Sequestration with Morbid Neonatal Pleural Effusion despite Successful Antenatal Treatment
}

\author{
Natalie Divjak ${ }^{1}$, Sabine Vasseur Maurer ${ }^{1}$, Eric Giannoni ${ }^{2}$, Yvan Vial $^{3}$, \\ Anthony de Buys Roessingh ${ }^{1}$ and Barbara E. Wildhaber ${ }^{1 *}$

\begin{abstract}
'Department Woman-Mother-Child, University Center of Pediatric Surgery of Western Switzerland, Lausanne University Hospital, Lausanne, Switzerland, '2Department Woman-Mother-Child, Clinic of Neonatology, Lausanne University Hospital, Lausanne, Switzerland, ${ }^{3}$ Department Woman-Mother-Child, Clinic of Obstetrics, Lausanne University Hospital, Lausanne, Switzerland
\end{abstract}

OPEN ACCESS

Edited by:

Offer Erez,

Soroka Medical Center, Israel

Reviewed by:

Ruben Witlox

Leiden University, Netherlands

Salvatore Andrea Mastrolia, Università degli studi di

Bari Aldo Moro, Italy

Orna Staretz Chacham,

Ben-Gurion University of the Negev, Israel

${ }^{*}$ Correspondence:

Barbara E. Wildhaber

Barabara.Wildhaber@chuv.ch

Specialty section: This article was submitted to Neonatology,

a section of the journal

Frontiers in Pediatrics

Received: 10 September 2017 Accepted: 20 November 2017 Published: 04 December 2017

Citation:

Divjak N, Vasseur Maurer S, Giannoni $E$, Vial $Y$,

de Buys Roessingh A and Wildhaber BE (2017)

Bronchopulmonary Sequestration with Morbid Neonatal Pleural

Effusion despite Successful Antenatal Treatment.

Front. Pediatr. 5:259.

doi: 10.3389/fped.2017.00259
Introduction: Bronchopulmonary sequestration (BPS) may cause prenatal pleural effusion (PE) or even hydrops. This case describes a fetus presenting with severe PE, which prenatally waned completely under steroid treatment, yet surprisingly reappeared rapidly after birth, requiring early surgical intervention.

Case description: A male fetus was diagnosed with left BPS and severe PE. After three courses of prenatal steroid therapy for each recurrence of PE from 27 weeks of gestation, we observed a complete regression of PE prenatally. Yet, PE recurred $18 \mathrm{~h}$ after birth and persisted after repeated drainages and steroid therapy. Early total resection of the extralobar BPS was performed and led to complete recovery without recurrence of PE.

Conclusion: This report underlines that in cases of BPS presenting with prenatal PE needing fetal intervention, even if full regression of PE is observed before birth, there might be a need for surgical excision during the neonatal period.

Keywords: bronchopulmonary sequestration, pleural effusion, antenatal treatment, neonatology, pediatric surgery

\section{INTRODUCTION}

Routine ultrasound screening during pregnancy usually may allow for the early detection of bronchopulmonary malformations. Various congenital lung lesions may present with associated complications, such as pleural effusion (PE), mediastinal shift, hydrops, and polyhydramnios. In the case of bronchopulmonary sequestration (BPS), up to one-third of fetuses present with severe PE or hydrops and require prenatal intervention $(1,2)$. Among these complications, hydrops has been shown to be the single prenatal outcome predictor for mortality $(2,3)$. Perinatal mortality concerns about $10 \%$ of babies presenting with BPS (2). Of note, $17 \%$ of infants born with BPS will present symptoms requiring emergency surgery during the first month of life (4). However, most of the infants will develop symptoms only later on, with an average time of onset of symptoms in prenatally detected lung lesions of about 7 months, thus only needing elective surgery during early infancy (4).

\section{CASE REPORT}

A 26-week-old male fetus presented with a pulmonary mass of $19 \mathrm{~cm}^{3}$, seen at standard ultrasound screening in our tertiary university hospital. A feeding vessel originating from the aorta was visualized

Abbreviations: BPS, bronchopulmonary sequestration; PE, pleural effusion. 
and suggested the diagnosis of BPS. Weekly ultrasound monitoring was performed thereafter. At 27 weeks of gestation, moderate PE appeared, for which intramuscular betamethasone was administered to the mother. Ultrasound follow-up showed fluctuation of PE with good response to steroids at each recurrence. Three courses of betamethasone $2 \mathrm{mg}$ /day for 2 days were administrated in total at $275 / 7,312 / 7$, and $341 / 7$ weeks of gestation, with $\mathrm{PE}$ regression after each course. Amniodrainage was performed at 32 weeks for polyhydramnios (amniotic fluid index 44), because of maternal discomfort and risk of premature rupture of membranes, which yet recurred thereafter. At 34 weeks, when the thoracic lesion measured $23 \mathrm{~cm}^{3}$, scalp edema was observed concomitant with $\mathrm{PE}$, however, followed by its complete regression after the third administration of betamethasone. Prenatal course of PE is summarized in Figure 1.

At 35 5/7 weeks, premature rupture of membranes occurred, probably due to recurrence of polyhydramnios and previous drainage. Cardiotocogram during labor was without pathological findings and vaginal delivery was uncomplicated. The newborn adapted well with Apgar score of 8/10/10. Birth weight was $2,570 \mathrm{~g}$ (P10-P50). There was no sign of respiratory distress or cardiovascular compromise. A first X-ray performed $4 \mathrm{~h}$ after birth showed a left basal thoracic mass without significant PE (Figure 2A). Yet, the newborn developed respiratory distress at the age of $18 \mathrm{~h}$ requiring treatment with high flow nasal cannula. $\mathrm{X}$-ray showed massive left sided PE causing mediastinal shift (Figure 2B). Immediate pleural drainage allowed for clinical improvement. The analysis of the pleural fluid showed low protein and low leukocyte content compatible with transudate.

Persistent active pleural drainage during the following days lead to radiological work-up by ultrasound and CT-scan confirming the diagnosis of extralobar BPS, measuring $3.9 \mathrm{~cm} \times 4.8 \mathrm{~cm} \times 3.9 \mathrm{~cm}$, with a feeding vessel originating from the aorta at the level of the diaphragm.

At 1 week of age, pleural drainage increased up to $80 \mathrm{ml} / \mathrm{kg}$ / day. Dexamethasone was administered for 1 week, at a decreasing dosage of $0.15 \mathrm{mg} / \mathrm{kg} /$ day for 3 days to $0.1 \mathrm{mg} / \mathrm{kg} /$ day, followed by complete resolution of $\mathrm{PE}$, and thus removal of the chest tube. One day after discontinuation of the steroid treatment, PE recurred

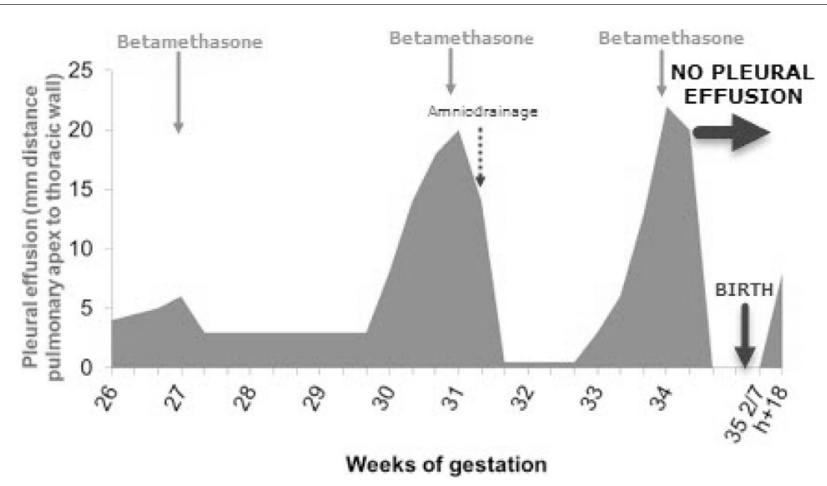

FIGURE 1 | Prenatal course of pleural effusion (PE) and its treatment. In total, three courses of betamethasone were administrated, allowing for complete prenatal regression of the $\mathrm{PE}$. needing a new chest tube insertion. Early excisional surgery was then considered as inevitable, and at day 27 the extralobar BPS was removed by thoracotomy. Histological analysis confirmed diagnosis of BPS and extensive pleuropulmonary lymphatic dilatation. Post-operative course was uneventful with complete recovery within days.

\section{DISCUSSION}

The antenatal discovery of a cystic or solid lung malformation opens a range of diagnosis, with the most frequent being congenital pulmonary airway malformation and BPS. Recent studies show that even the distinction between these two entities is not so clear with the discovery of an increasing number of "hybrid" lesions (3). Natural fetal evolution of these lesions may either tend toward enlargement, or to steady state, or even to partial/ complete regression (3). When presenting antenatal complications, such as massive PE, with or without mediastinal shift, or even hydrops, fetal intervention may be required (5). Pleuralamniotic shunting is an option in these cases. BPS offers more specific therapeutic options by acting on the feeding vessel by laser, radiofrequency, or embolization. A recent study comparing pleuroamniotic drainage to laser ablation of the feeding vessel showed a tendency toward an advantage for ablation of the feeding vessel, even though numbers are too small to demonstrate a definitive advantage (1). In the case of microcystic lesions, maternal steroid treatment with betamethasone has shown to be a safe and efficient by reducing hydrops and also the size of the lesions. Of note, no adverse maternal or fetal events resulting from betamethasone administration have been reported (6). It is believed that the administration of steroids allows for lung maturation in these bronchopulmonary malformations, which are considered as an arrested state of development. Yet, the role of maternal betamethasone treatment in BPS has not been clearly established due to their relative lower incidence (7). In our case, where steroid administration was chosen, the fetus responded to repeated courses of betamethasone with complete regression of $\mathrm{PE}$ and decrease in size of the BPS.

Because there is a twofold increase in risk of operative complications in symptomatic patients, elective surgery is aimed to be performed before appearance of symptoms, such as respiratory distress or infection (4). Average time of onset of symptoms is reported to be at the age of about 10 months, taking into consideration both infants diagnosed prenatally and those who were not (4). Thus, most pediatric surgeons generally plan elective surgery in infants presenting with congenital lung lesions at the age of 6-12 months (8). The reported neonate was completely asymptomatic at birth, and also once the postnatally recurrent $\mathrm{PE}$ was drained, allowing time for observation of natural evolution of the PE. In addition, we attempted a conservative treatment with corticosteroids, since this was effective during pregnancy. Response was favorable, but did not avoid recurrence of PE. We then chose to perform surgery to avoid prolonged pleural drainage, with the risk of infection, as well as long-term steroid treatment, associated with its well-known side effects.

To the best of our knowledge, the postnatal recurrence of $\mathrm{PE}$ after complete antenatal regression has not been described in 


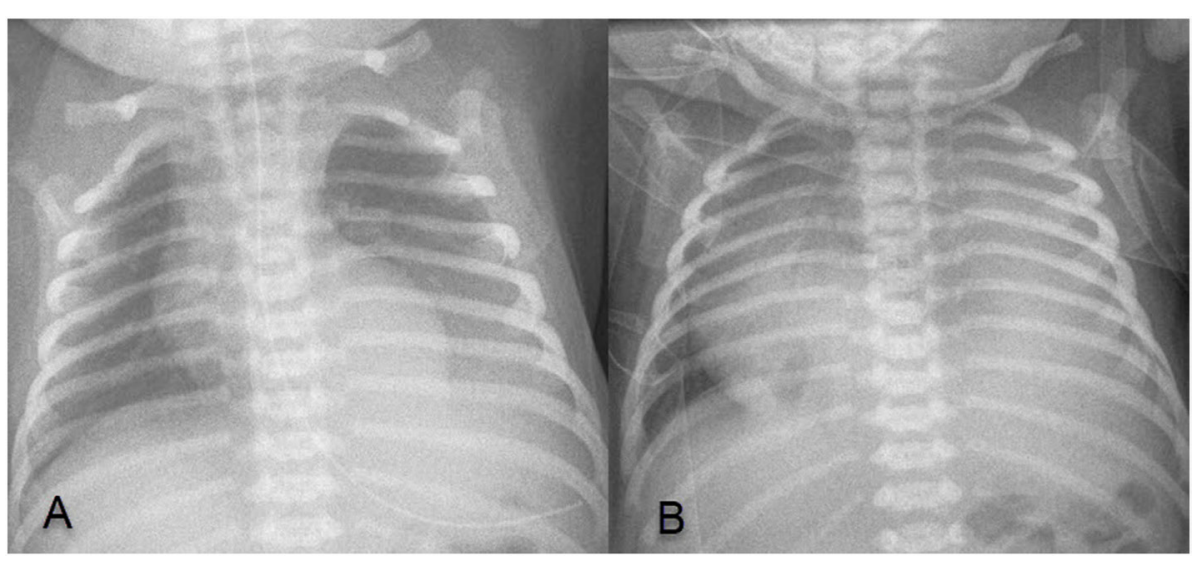

FIGURE 2 | (A) X-ray $4 \mathrm{~h}$ after birth showing left basal thoracic mass, without significant pleural effusion (PE); (B) X-ray at $18 \mathrm{~h}$ of age showing a massive left sided PE causing mediastinal shift.

the literature. The reason for postnatal recurrence of $\mathrm{PE}$ remains unclear. Reports suggest that severe PE might be due to obstruction of venous return due to compression by the BPS, cardiac failure due to shunting through the lesion or obstruction of lymphatic return. The chemical and cytological analysis of the pleural liquid, compatible with transudate, is in line with this hypothesis. Histological examination in our case showed marked lymphatic dilatation, a potential explanation for recurrent PE in our case: steroids may have influenced the local lymphatic tissue, yet without persistent effect.

\section{CONCLUDING REMARKS}

Antenatal ultrasound scan is a sensitive tool, which offers early detection of BPS and its antenatal complications, and thus guides intervention. These measures may lead to complete antenatal

\section{REFERENCES}

1. Mallmann MR, Geipel A, Bludau M, Matil K, Gottschalk I, Hoopmann M, et al. Bronchopulmonary sequestration with massive pleural effusion: pleuroamniotic shunting vs intrafetal vascular laser ablation. Ultrasound Obstet Gynecol (2014) 44:441-6. doi:10.1002/uog.13304

2. Stoiber B, Moehrlen U, Kurmanavicius J, Meuli M, Haslinger C, Zimmermann R, et al. Congenital lung lesion: prenatal course, therapy and predictors of perinatal outcome. Ultraschall Med (2015) 38(02):158-65. doi:10.1055/s-00351553261

3. Davenport M, Warne SA, Cacciaguerra S, Patel S, Greenough A, Nicolaides K. Current outcome of antenally diagnosed cystic lung disease. J Pediatr Surg (2004) 39:549-56. doi:10.1016/j.jpedsurg.2003.12.021

4. Stanton M, Njere I, Ade-Ajayi N, Patel S, Davenport M. Systematic review and meta-analysis of the postnatal management of congenital cystic lung lesions. J Pediatr Surg (2009) 44:1027-33. doi:10.1016/j.jpedsurg.2008.10.118

5. Hellmund A, Berg C, Geipel A, Bludau M, Heydweiller A, Bachour H, et al. Prenatal diagnosis and evaluation of sonographic predictors for intervention and adverse outcome in congenital pulmonary airway malformation. PLoS One (2016) 11(3):e0150474. doi:10.1371/journal.pone.0150474

6. Downard CD, Calkins CM, Williams RF, Renaud EJ, Jancelewicz T, Grabowski J, et al. Treatment of congenital pulmonary airway malformations: a systematic resolution of PE. Nevertheless, this case illustrates that, despite a favorable antenatal course, there is a probability that surgical intervention during the neonatal period still might be required due to recurrence of $\mathrm{PE}$ after birth.

\section{ETHICS STATEMENT}

Parental written consent was obtained.

\section{AUTHOR CONTRIBUTIONS}

ND and BW were responsible for the initial written content as well as editing the manuscript and creating the image. All other authors were responsible for reviewing and editing the manuscript in its intellectual content as well as its form; gave their final approval for the version to be published.

review from the APSA outcomes and evidence based practice committee Pediatr Surg Int (2017) 33:939. doi:10.1007/s00383-017-4098-Z

7. Peranteau WH, Boelig MM, Khalek N, Moldenhauer JS, Martinez-Poyer J, Hedrick HL, et al. Effect of single and multiple courses of maternal betamethasone on prenatal congenital lung lesion growth and fetal survival. J Pediatr Surg (2016) 51:28-32. doi:10.1016/j.jpedsurg.2015.10.018

8. David M, Lamas-Pinheiro R, Henriques-Coelho T. Prenatal and postnatal management of congenital pulmonary airway malformation. Neonatology (2016) 110:101-15. doi:10.1159/000440894

Conflict of Interest Statement: The authors declare that the research was conducted in the absence of any commercial or financial relationships that could be construed as a potential conflict of interest.

Copyright (C) 2017 Divjak, Vasseur Maurer, Giannoni, Vial, de Buys Roessingh and Wildhaber. This is an open-access article distributed under the terms of the Creative Commons Attribution License (CC BY). The use, distribution or reproduction in other forums is permitted, provided the original author(s) or licensor are credited and that the original publication in this journal is cited, in accordance with accepted academic practice. No use, distribution or reproduction is permitted which does not comply with these terms. 